\title{
Mitochondrial Respiration-Improving Effects of Three Different Gardeniae Fructus Preparations and Their Components
}

Yun Wang ${ }^{1 \dagger}$, Puling $\mathrm{Li}^{1,2 \uparrow}$, Xue Zhang ${ }^{1 \dagger}$, Lingyun $\mathrm{Li}^{1,2}$, Mengjiao Liu ${ }^{1}$, Xiaoqing $\mathrm{Li}^{1,2}$, Yejia

Dai $^{1}$, Cun Zhang ${ }^{1,2^{*}}$, Shaojing $\mathrm{Li}^{1 *}$

1. Institute of Chinese Materia Medica, China Academy of Chinese Medical Sciences, Beijing 100700, China;

2. School of Pharmacy, Henan University of TCM, Zhengzhou 450008, China;

+ Yun Wang, Puling Li and Xue Zhang are coauthors.

*Corresponding authors:

\section{Cun Zhang}

Institute of Chinese Materia Medica, China Academy of Chinese Medical Sciences, Beijing 100700, China, Email: zhc95@163.com

\section{Shao-Jing Li}

Institute of Chinese Materia Medica, China Academy of Chinese Medical Sciences, Beijing 100700, China, Email: shaojingli2004@126.com

*These authors contributed equally to this work.

\section{Contents}

Figure S1. Chemometric analysis of HPLC fingerprints of three GF preparations. (A) Cluster analysis; (B) PLS-DA analysis. Green color refers to raw GF; Blue and red color refer to GFP and GFC; Yellow refers to GFP and GFC.

Figure S2. Parameters of mitochondrial respiration in each group (GF, GFP and GFC at a dose of $0.5 \mathrm{~g} \cdot \mathrm{kg}-1$ ), including state 3 respiration (A), state 4 respiration (B), RCR (C), OPR (D), and ADP/O ratio (E). Glutamate $(10 \mathrm{mmol} / \mathrm{L})$ and malate $(5 \mathrm{mmol} / \mathrm{L})$ were used as respiration substrates. Oxygen consumption is expressed in $\mathrm{nmol} \mathrm{O} 2 /$ min per $\mathrm{mg}$ protein (mean $\pm \mathrm{SD}$ ). $\# \mathrm{P}<0.05$ compared with sham; $* \mathrm{P}<0.05$ compared with model group.

Figure S3. Effects of GF, GFP and GFC on mitochondrial respiratory function in MCAO rats, evaluated by PCA analysis. (A) at a drug dose of $1 \mathrm{~g} \cdot \mathrm{kg}^{-1}$ and (B) at a drug dose of $0.5 \mathrm{~g} \cdot \mathrm{kg}^{-1}$. Values are presented as the mean $\pm \mathrm{SD}$ of six rats. $\mathrm{S}=\mathrm{GF}, \mathrm{J}=\mathrm{GFP}$, and $\mathrm{T}=\mathrm{GFC}, p<0.01$ model group vs. the sham group; $p<0.05, p<0.01$, drug-treated groups vs. the model group (one-way ANOVA). Figure S4. GF, GFP and GFC decreased the mitochondrial ROS production in MCAO model at the dose of $1 \mathrm{~g} \cdot \mathrm{kg}-1$. ROS was determined spectrofluorometrically by using the fluorescent dye DCFH. All data are expressed means $\pm \mathrm{SD}, \mathrm{M}$ indicates model group, Ed indicates edaravone. $\mathrm{S}$ indicated GF, J indicates GFP, $\mathrm{T}$ indicates GFC, \#\#P<0.01vs Model group $* * P<0.01,{ }^{*} p<0.05$ drugs treated group vs. the model group. Significance was determined by one-way ANOVA.

Figure S5. Geniposide, crocin and GC3, attenuated neurological deficits induced by MCAO in rats. 
Values are expressed as the mean $\pm \mathrm{SD}(\mathrm{n}=6)$, and the data were analyzed by one-way ANOVA. $\mathrm{G}$ indicated geniposide, $\mathrm{C}$ indicates crocin, Eg indicated Egb761, M indicates model group, GC1 indicates crocin: geniposide $=1: 3, \mathrm{GC} 2$ indicates crocin: geniposide $=1: 1 \mathrm{GC} 3$ indicates crocin: geniposide=3:1. \#\#P<0.01 vs. Model group, ${ }^{* *} P<0.01,{ }^{*} p<0.05$ drugs treated group vs. the model group.

Figure S6. Effects of GC3 on neurological deficits induced by MCAO in rats. (A) TTC stained coronal sections of rat brains $(\mathrm{n}=6)$. Red and white colors denote the normal and infarcted areas, respectively. The colorless region corresponds to the occluded middle cerebral artery territory. (B) Infarct volumes in different groups, determined by TTC staining and photographic image analysis. Values are expressed as the mean \pm standard deviation (SD, $\mathrm{n}=6$ ), ${ }^{\#} p<0.01$ model group vs. the sham group; ${ }^{* *} p<0.05,{ }^{*} p<0.01$ drug-treated groups vs. the model group (one-way ANOVA), M =MCAO model group; $\mathrm{Ed}=$ edaravone.

Figure S7. Crocin, geniposide and GC3 improved mitochondrial respiration function in MCAO rats by PCA analysis. Values are presented as the mean $\pm \mathrm{SD}$ of six rats and were analyzed by one-way ANOVA. $\mathrm{G}$ indicated geniposide, $\mathrm{C}$ indicates crocin, $\mathrm{Ed}$ indicates edaravone, $\mathrm{M}$ indicates model group, $\mathrm{GC} 1$ indicates crocin: geniposide $=1: 3, \mathrm{GC} 2$ indicates crocin: geniposide $=1: 1 \mathrm{GC} 3$ indicates crocin: geniposide $=3: 1 . \# \# P<0.01$ vs. Model group $* * P<0.01$,

$*_{p}<0.05$ drugs treated group vs. the model group.

Figure S8. Crocin was more important than geniposide on mitochondrial respiration function by variable importance in projection (VIP) analysis. VIP $>1$ was selected as the cut off value to identify the most important variable.

Table S1. The iridoid glycoside content of crude GF $(\mathrm{mg} / \mathrm{g})$.

Table S2. The iridoid glycoside content of stir-baked GF $(\mathrm{mg} / \mathrm{g})$.

Table S3. The iridoid glycoside content of carbonized GF $(\mathrm{mg} / \mathrm{g})$.

Table S4. Table S4. The diterpenoid content of processed GF (mg/g).

A

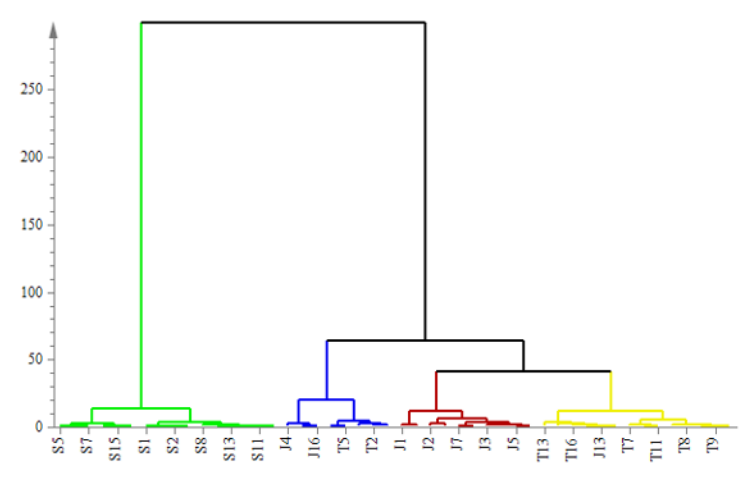

B

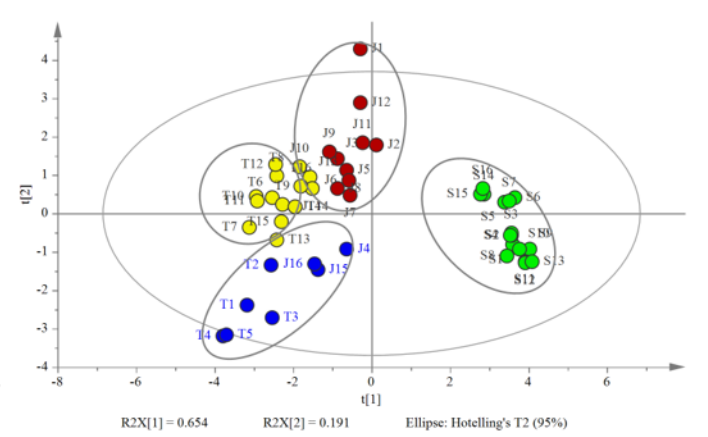

Figure S1. Chemometric analysis of HPLC fingerprints of three GF preparations. (A) Cluster analysis; (B) PLS-DA analysis. Green color refers to raw GF; Blue and red color refer to GFP and GFC; Yellow refers to GFP and GFC. 

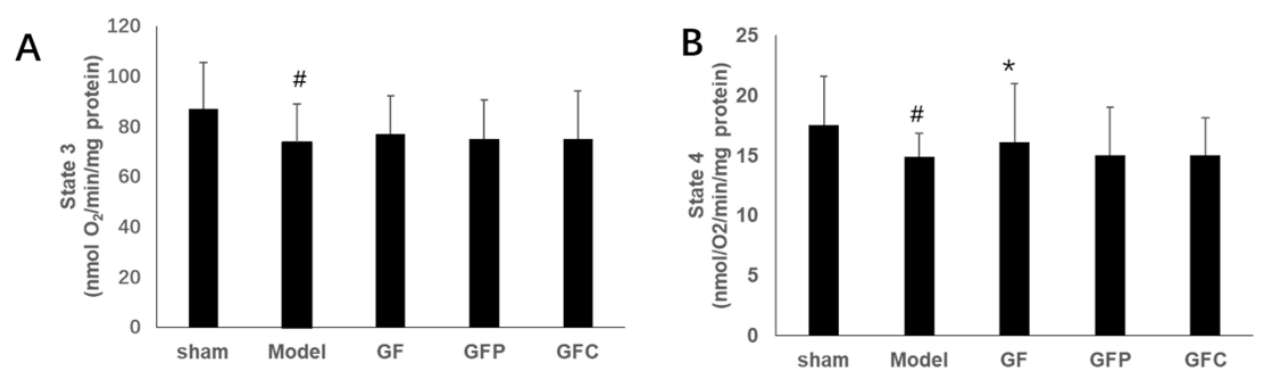

C
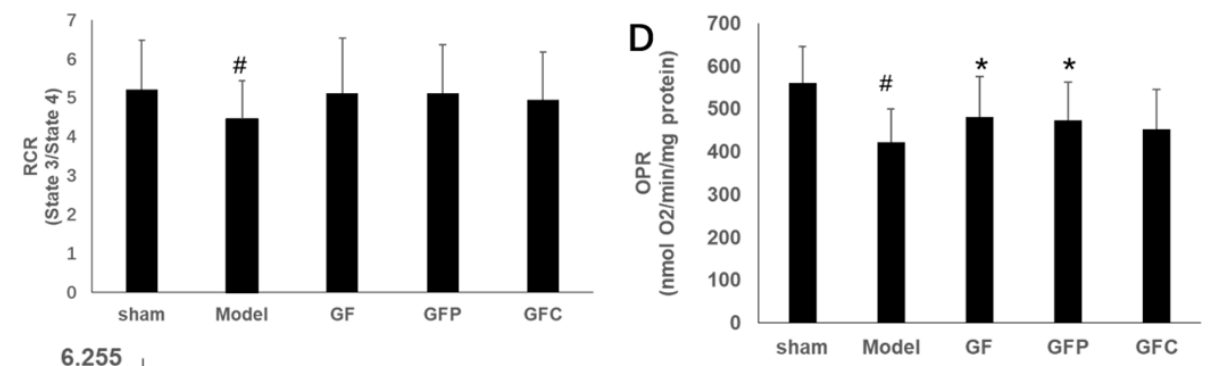

$\mathrm{E}$

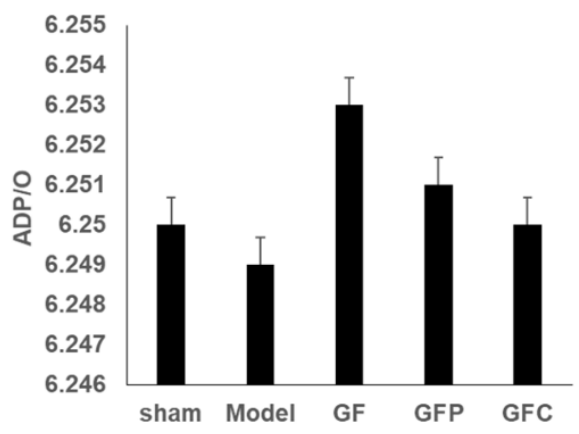

Figure S2. Parameters of mitochondrial respiration in each group (GF, GFP and GFC at a dose of $0.5 \mathrm{~g} \cdot \mathrm{kg}^{-1}$ ), including state 3 respiration (A), state 4 respiration (B), RCR (C), OPR (D), and ADP/O ratio $(\mathrm{E})$. Glutamate $(10 \mathrm{mmol} / \mathrm{L})$ and malate $(5 \mathrm{mmol} / \mathrm{L})$ were used as respiration substrates. Oxygen consumption is expressed in nmol O2/min per mg protein (mean $\pm \mathrm{SD}$ ). ${ }^{\#} P<0.05$ compared with sham; ${ }^{*} P<0.05$ compared with model group.
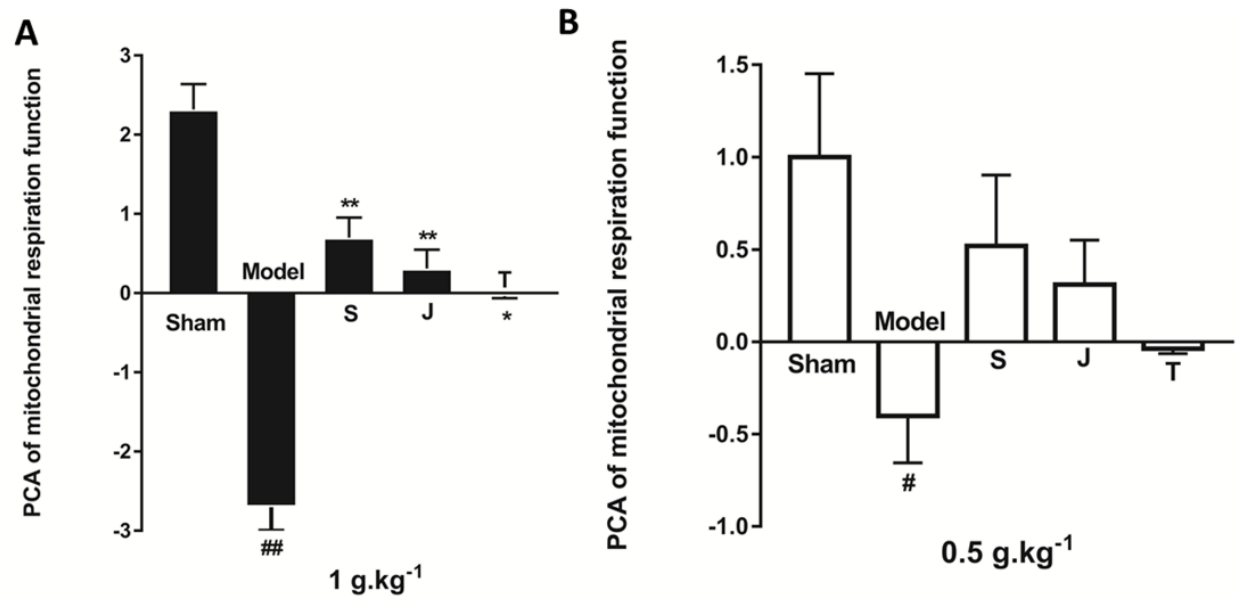

Figure S3. Effects of GF, GFP and GFC on mitochondrial respiratory function in MCAO rats, evaluated by PCA. (A) at a drug dose of $1 \mathrm{~g} \cdot \mathrm{kg}^{-1}$ and (B) at a drug dose of $0.5 \mathrm{~g} \cdot \mathrm{kg}^{-1}$. Values are presented as the mean $\pm \mathrm{SD}$ of six rats. $\mathrm{S}=\mathrm{GF}, \mathrm{J}=\mathrm{GFP}$, and $\mathrm{T}=\mathrm{GFC}, p<0.01$ model group vs. the sham group; $p<0.05, p<0.01$, drug-treated groups vs. the model group (one-way ANOVA). 


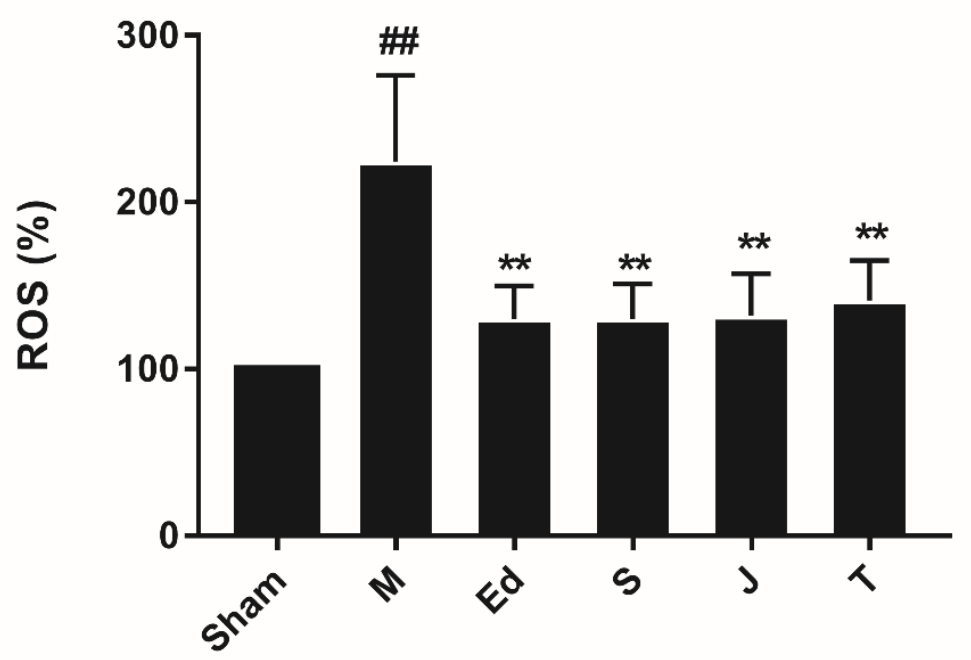

Figure S4. GF, GFP and GFC decreased the mitochondrial ROS production in MCAO model at the dose of $1 \mathrm{~g} \cdot \mathrm{kg}-1$. ROS was determined spectrofluorometrically by using the fluorescent dye DCFH. All data are expressed means $\pm \mathrm{SD}, \mathrm{M}$ indicates model group, Ed indicates edaravone. $\mathrm{S}$ indicated GF, J indicates GFP, $\mathrm{T}$ indicates GFC, \#\#P<0.01vs Model group $* * P<0.01,{ }^{*} p<0.05$ drugs treated group vs the model group. Significance was determined by one-way ANOVA.

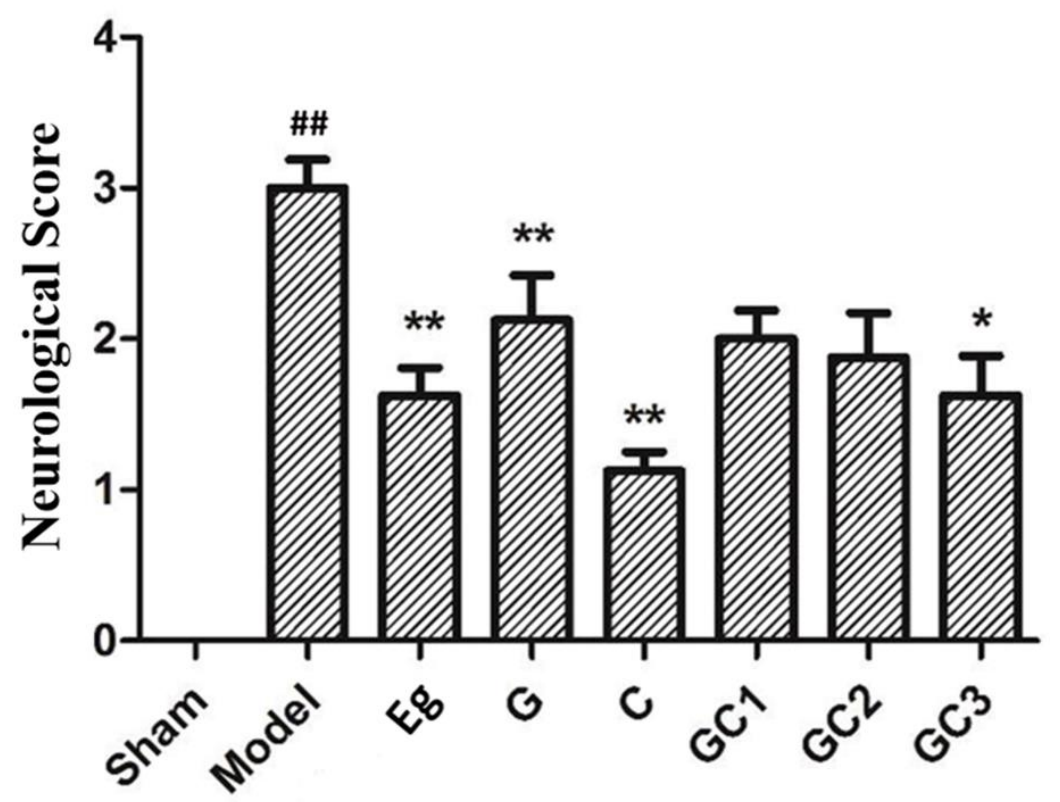

Figure S5. Geniposide, crocin and GC3, attenuated neurological deficits induced by MCAO in rats. Values are expressed as the mean $\pm \mathrm{SD}(\mathrm{n}=6)$, and the data were analyzed by one-way ANOVA. $\mathrm{G}$ indicated geniposide, $\mathrm{C}$ indicates crocin, Eg indicated Egb761, M indicates model group, GC1 indicates crocin: geniposide $=1: 3, \mathrm{GC} 2$ indicates crocin: geniposide $=1: 1 \mathrm{GC} 3$ indicates crocin: geniposide=3:1. \#\#P<0.01 vs Model group, ${ }^{* *} P<0.01,{ }^{*} p<0.05$ drugs treated group vs the model group. 

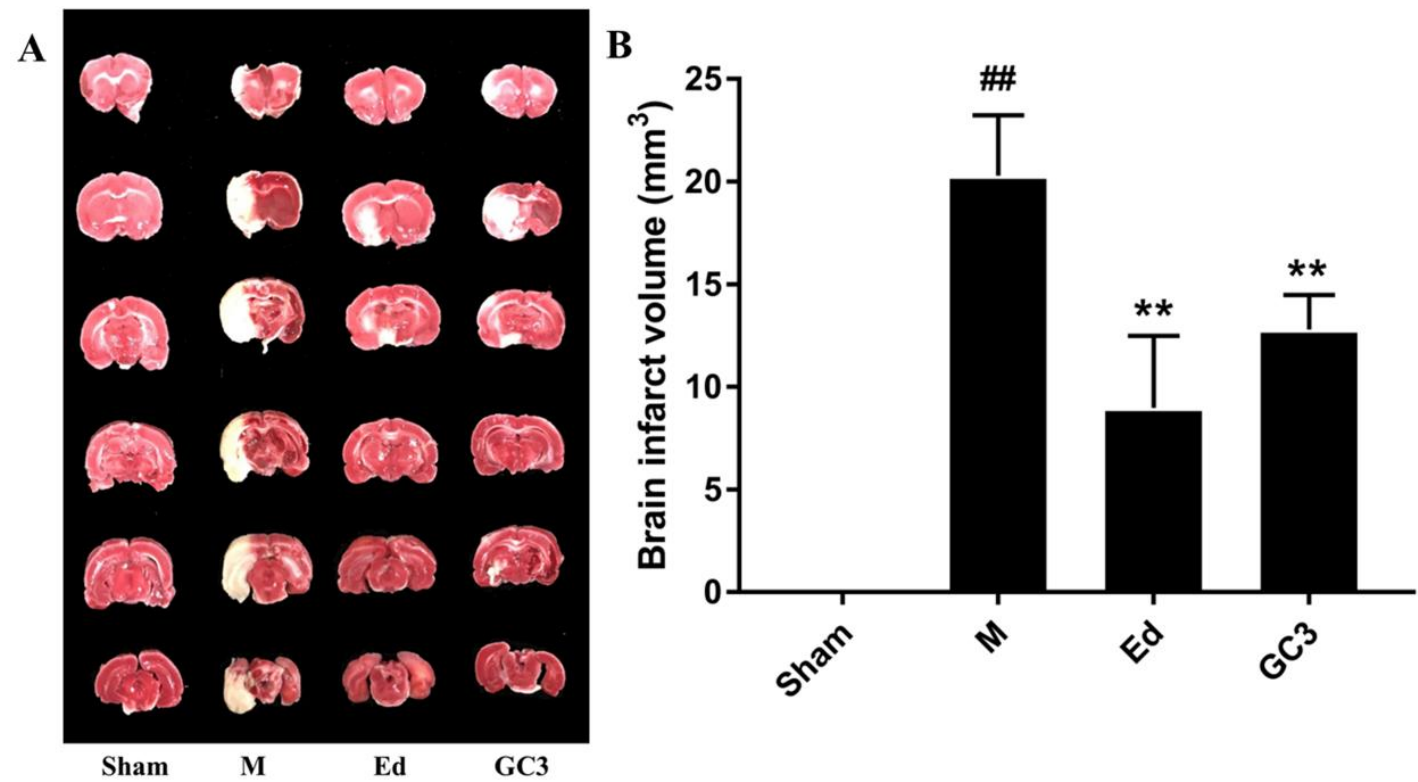

Figure S6. Effects of GC3 on neurological deficits induced by MCAO in rats. (A) TTC stained coronal sections of rat brains $(\mathrm{n}=6)$. Red and white colors denote the normal and infarcted areas, respectively. The colorless region corresponds to the occluded middle cerebral artery territory. (B) Infarct volumes in different groups, determined by TTC staining and photographic image analysis. Values are expressed as the mean \pm standard deviation (SD, $\mathrm{n}=6$ ), ${ }^{\#} p<0.01$ model group vs the sham group; ${ }^{*} p<0.05,{ }^{*} p<0.01$ drug-treated groups vs. the model group (one-way ANOVA). M $=\mathrm{MCAO}$ model group; $\mathrm{Ed}=$ edaravone.

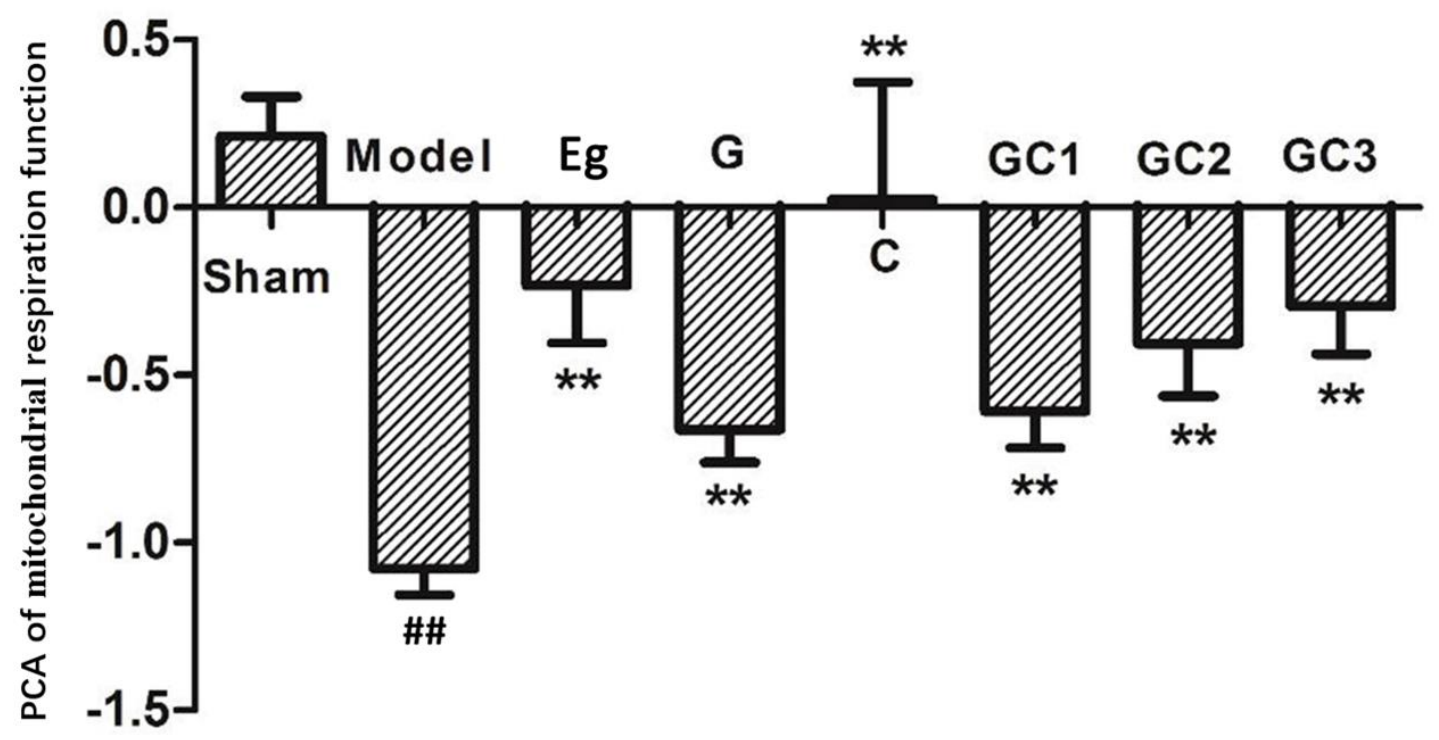

Figure S7. Crocin, geniposide and GC3 improved mitochondrial respiration function in MCAO rats by PCA analysis. Values are presented as the mean $\pm \mathrm{SD}$ of six rats and were analyzed by one-way ANOVA. $\mathrm{G}$ indicated geniposide, $\mathrm{C}$ indicates crocin, $\mathrm{Ed}$ indicates edaravone, $\mathrm{M}$ indicates model group, $\mathrm{GC} 1$ indicates crocin: geniposide $=1: 3, \mathrm{GC} 2$ indicates crocin: geniposide=1:1 GC3 indicates crocin:geniposide $=3: 1 . \# \# P<0.01$ vs Model group $* * P<0.01$, ${ }^{*} p<0.05$ drugs treated group versus the model group. 


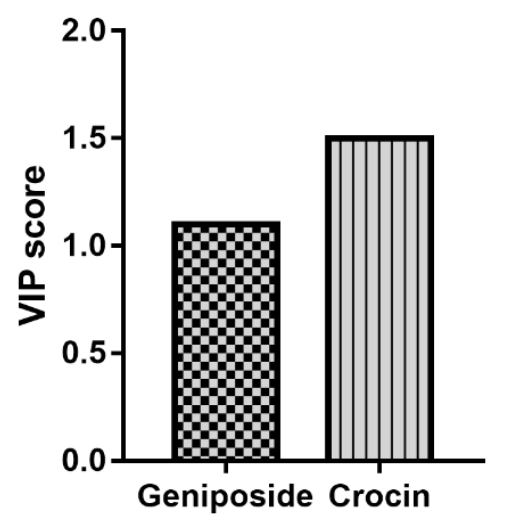

Figure S8. Crocin was more important than geniposide on mitochondrial respiration function by variable importance in projection (VIP) analysis. VIP $>1$ was selected as the cut off value to identify the most important variable.

Table S1. The iridoid glycoside content of crude GF (mg/g).

\begin{tabular}{cccccccccc}
\hline number & $\mathrm{a}$ & $\mathrm{b}$ & $\mathrm{c}$ & $\mathrm{d}$ & $\mathrm{e}$ & $\mathrm{f}$ & $\mathrm{h}$ & $\mathrm{i}$ & Total \\
\hline S1 & 2.82 & 0.53 & 0.79 & 7.42 & 0.51 & 22.70 & 55.29 & 5.66 & 95.71 \\
S2 & 2.83 & 0.49 & 0.70 & 7.33 & 0.50 & 23.43 & 56.90 & 5.54 & 97.73 \\
S3 & 2.83 & 0.48 & 0.70 & 7.18 & 0.50 & 23.43 & 57.03 & 5.55 & 97.69 \\
S4 & 2.82 & 0.50 & 0.70 & 7.27 & 0.51 & 23.48 & 57.04 & 5.55 & 97.88 \\
S5 & 2.79 & 0.50 & 0.75 & 7.01 & 0.47 & 25.68 & 57.54 & 5.98 & 100.72 \\
S6 & 2.84 & 0.46 & 0.76 & 6.36 & 0.42 & 25.81 & 57.39 & 6.01 & 100.05 \\
S7 & 2.68 & 0.47 & 0.79 & 6.67 & 0.42 & 25.77 & 57.28 & 6.01 & 100.09 \\
S8 & 2.94 & 0.58 & 0.78 & 6.39 & 0.45 & 22.20 & 58.80 & 5.77 & 97.92 \\
S9 & 3.03 & 0.53 & 0.82 & 6.31 & 0.44 & 22.29 & 58.85 & 5.78 & 98.05 \\
S10 & 2.78 & 0.51 & 0.83 & 6.13 & 0.42 & 22.28 & 58.54 & 5.77 & 97.26 \\
S11 & 2.80 & 0.50 & 0.81 & 7.08 & 0.42 & 21.63 & 58.48 & 5.56 & 97.27 \\
S12 & 2.82 & 0.50 & 0.81 & 7.07 & 0.41 & 21.66 & 58.62 & 5.56 & 97.44 \\
S13 & 3.00 & 0.49 & 0.81 & 7.10 & 0.40 & 21.68 & 58.73 & 5.55 & 97.76 \\
S14 & 2.40 & 0.46 & 0.70 & 6.37 & 0.38 & 26.33 & 54.46 & 6.05 & 97.14 \\
S15 & 2.50 & 0.48 & 0.67 & 6.34 & 0.42 & 26.24 & 54.73 & 6.02 & 97.40 \\
S16 & 2.52 & 0.46 & 0.61 & 5.95 & 0.46 & 26.14 & 54.67 & 6.02 & 96.84 \\
mean & 2.77 & 0.50 & 0.75 & 6.75 & 0.45 & 23.80 & 57.15 & 5.77 & 97.94 \\
\hline
\end{tabular}


Table S2. The iridoid glycoside content of stir-baked GF(mg/g).

\begin{tabular}{|c|c|c|c|c|c|c|c|c|c|}
\hline number & $\mathrm{a}$ & b & $\mathrm{c}$ & d & $\mathrm{e}$ & $\mathrm{f}$ & $\mathrm{h}$ & $\mathrm{i}$ & Total \\
\hline $\mathrm{J} 1$ & 2.61 & 0.43 & 0.36 & 0.98 & 0.43 & 30.86 & 48.00 & 7.67 & 91.34 \\
\hline $\mathrm{J} 2$ & 2.91 & 0.45 & 0.39 & 0.97 & 0.46 & 25.14 & 50.94 & 6.35 & 87.61 \\
\hline $\mathrm{J} 4$ & 2.98 & 0.65 & 0.35 & 0.51 & 0.43 & 21.61 & 53.89 & 5.10 & 85.52 \\
\hline J5 & 2.60 & 0.54 & 0.40 & 0.97 & 0.46 & 25.66 & 49.79 & 5.82 & 86.25 \\
\hline $\mathrm{J} 7$ & 2.81 & 0.64 & 0.41 & 1.07 & 0.42 & 24.87 & 51.26 & 5.88 & 87.36 \\
\hline J8 & 2.76 & 0.69 & 0.38 & 1.06 & 0.39 & 25.82 & 50.60 & 6.13 & 87.82 \\
\hline J9 & 2.48 & 0.45 & 0.37 & 0.74 & 0.35 & 25.36 & 46.95 & 6.25 & 82.94 \\
\hline $\mathrm{J} 10$ & 2.37 & 0.62 & 0.35 & 0.73 & 0.33 & 26.08 & 45.47 & 6.45 & 82.40 \\
\hline $\mathrm{J} 14$ & 2.47 & 0.60 & 0.39 & 0.81 & 0.38 & 24.43 & 42.99 & 6.07 & 78.13 \\
\hline $\mathrm{J} 15$ & 2.62 & 0.66 & 0.39 & 0.88 & 0.43 & 21.51 & 47.69 & 4.52 & 78.70 \\
\hline $\mathrm{J} 16$ & 2.57 & 0.66 & 0.37 & 0.86 & 0.42 & 21.88 & 47.95 & 4.63 & 79.35 \\
\hline mean & 2.62 & 0.57 & 0.38 & 0.89 & 0.41 & 25.42 & 48.96 & 6.02 & 85.28 \\
\hline
\end{tabular}

Table S3. The iridoid glycoside content of carbonized GF(mg/g).

\begin{tabular}{cccccccccc}
\hline number & $\mathrm{a}$ & $\mathrm{b}$ & $\mathrm{c}$ & $\mathrm{d}$ & $\mathrm{e}$ & $\mathrm{f}$ & $\mathrm{h}$ & $\mathrm{i}$ & Total \\
\hline $\mathrm{T} 1$ & 2.26 & 0.73 & 0.21 & 0.28 & 0.19 & 21.39 & 46.76 & 4.24 & 76.07 \\
T2 & 2.44 & 0.61 & 0.21 & 0.39 & 0.21 & 21.61 & 44.64 & 4.83 & 74.93 \\
T3 & 2.55 & 0.63 & 0.20 & 0.33 & 0.21 & 18.15 & 45.39 & 4.26 & 71.71 \\
T4 & 2.13 & 0.65 & 0.16 & 0.21 & 0.15 & 18.52 & 40.28 & 3.75 & 65.85 \\
T5 & 2.19 & 0.67 & 0.16 & 0.20 & 0.14 & 19.08 & 40.69 & 3.74 & 66.86 \\
T6 & 2.38 & 0.55 & 0.23 & 0.29 & 0.19 & 25.00 & 42.10 & 5.65 & 76.39 \\
T7 & 1.90 & 0.55 & 0.20 & 0.35 & 0.18 & 23.99 & 42.20 & 5.07 & 74.43 \\
T8 & 2.22 & 0.49 & 0.23 & 0.37 & 0.18 & 26.57 & 43.51 & 5.62 & 79.19 \\
T9 & 2.36 & 0.49 & 0.23 & 0.37 & 0.18 & 24.54 & 44.26 & 5.33 & 77.76 \\
T10 & 2.09 & 0.55 & 0.20 & 0.32 & 0.29 & 26.72 & 38.36 & 5.02 & 73.55 \\
T11 & 2.13 & 0.56 & 0.20 & 0.31 & 0.29 & 26.35 & 38.48 & 5.01 & 73.33 \\
T12 & 2.08 & 0.61 & 0.22 & 0.49 & 0.32 & 28.12 & 44.86 & 5.85 & 82.54 \\
T13 & 2.19 & 0.65 & 0.22 & 0.47 & 0.33 & 23.85 & 46.69 & 4.94 & 79.34 \\
T14 & 2.42 & 0.54 & 0.24 & 0.45 & 0.32 & 24.33 & 44.96 & 5.27 & 78.51 \\
T15 & 2.38 & 0.52 & 0.26 & 0.43 & 0.19 & 23.92 & 44.06 & 5.08 & 76.83 \\
T16 & 2.50 & 0.60 & 0.25 & 0.58 & 0.23 & 25.80 & 50.01 & 6.03 & 86.00 \\
mean & 2.26 & 0.59 & 0.21 & 0.36 & 0.22 & 23.62 & 43.58 & 4.98 & 75.83 \\
\hline
\end{tabular}


Table S4. The diterpenoid content of processed GF (mg/g)

\begin{tabular}{cccccccccc}
\hline number & Sheng-j & Sheng-k & Sheng-t & Jiao-j & Jiao-k & Jiao-t & Tan-j & Tan-k & Tan-t \\
\hline S1 & 10.87 & 1.48 & 12.36 & 0.58 & 0.04 & 0.62 & 0.05 & 0.00 & 0.05 \\
S2 & 10.88 & 1.49 & 12.36 & 0.66 & 0.05 & 0.71 & 0.03 & 0.00 & 0.03 \\
S3 & 10.89 & 1.49 & 12.38 & 0.27 & 0.02 & 0.29 & 0.03 & 0.00 & 0.03 \\
S4 & 10.90 & 1.49 & 12.39 & 0.31 & 0.02 & 0.33 & 0.01 & 0.00 & 0.01 \\
S5 & 10.63 & 1.47 & 12.10 & 0.16 & 0.01 & 0.17 & 0.01 & 0.00 & 0.01 \\
S6 & 10.69 & 1.47 & 12.16 & 0.17 & 0.01 & 0.18 & 0.02 & 0.00 & 0.02 \\
S7 & 10.69 & 1.47 & 12.16 & 0.49 & 0.03 & 0.52 & 0.02 & 0.00 & 0.02 \\
S8 & 11.38 & 1.48 & 12.86 & 0.50 & 0.03 & 0.53 & 0.07 & 0.00 & 0.08 \\
S9 & 11.41 & 1.49 & 12.90 & 0.21 & 0.01 & 0.22 & 0.07 & 0.00 & 0.08 \\
S10 & 11.40 & 1.49 & 12.89 & 0.19 & 0.01 & 0.20 & 0.04 & 0.00 & 0.04 \\
S11 & 11.59 & 1.51 & 13.10 & 0.80 & 0.05 & 0.85 & 0.04 & 0.00 & 0.04 \\
S12 & 11.59 & 1.51 & 13.11 & 0.81 & 0.05 & 0.86 & 0.06 & 0.00 & 0.06 \\
S13 & 11.61 & 1.52 & 13.13 & 0.12 & 0.01 & 0.13 & 0.07 & 0.00 & 0.08 \\
S14 & 10.34 & 1.36 & 11.70 & 0.12 & 0.01 & 0.13 & 0.11 & 0.01 & 0.12 \\
S15 & 10.30 & 1.36 & 11.66 & 0.17 & 0.01 & 0.18 & 0.11 & 0.01 & 0.12 \\
S16 & 10.29 & 1.36 & 11.65 & 0.16 & 0.01 & 0.17 & 0.10 & 0.01 & 0.11 \\
mean & 10.97 & 1.47 & 12.43 & 0.36 & 0.02 & 0.38 & 0.05 & 0.00 & 0.06 \\
\hline
\end{tabular}

